\title{
Inversion of spherical means and the wave equation in even dimensions
}

\author{
David Finch \\ Department of Mathematics \\ Oregon State University \\ Corvallis, OR 97331 \\ finch@math.oregonstate.edu
}

\author{
Markus Haltmeier \\ Department of Computer Science \\ Universität Innsbruck \\ Technikerstraße 21a \\ A-6020 Innsbruck, Austria \\ Markus.Haltmeier@uibk.ac.at
}

\author{
Rakesh \\ Department of Mathematical Sciences \\ University of Delaware \\ Newark, DE 19716 \\ rakesh@math.udel.edu
}

January 15, 2007

Keywords: spherical means, wave equation, thermoacoustic tomography

AMS subject classifications: 35R30, 35L05, 35Q05, 92C55, 65R32

\begin{abstract}
We establish inversion formulas of the so called filtered back-projection type to recover a function supported in the ball in even dimensions from its spherical means over spheres centered on the boundary of the ball. We also find several formulas to recover initial data of the from $(f, 0)$ (or $(0, g))$ for the free space wave equation in even dimensions from the trace of the solution on the boundary of the ball, provided the initial data has support in the ball.
\end{abstract}




\section{Introduction and Statement of Results}

The problem of determining a function from a subset of its spherical means has a rich history in pure and applied mathematics. Our interest in the subject was provoked by the new medical imaging technologies called thermoacoustic and photoacoustic tomography. The idea behind these [10, 16] is to illuminate an object by a short burst of radiofrequency or optical energy which causes rapid (though small in magnitude) thermal expansion which generates an acoustic wave. The acoustic wave can be measured on the periphery or in the exterior of the object. The inverse problem we consider is to find the distribution of the absorbed energy throughout the body. This is of interest, since the amount of energy absorbed at different points may be diagnostic of disease or indicative of uptake of probes tagged to metabolic processes or gene expression [9]. For a more thorough discussion of the modelling and biomedical applications, the reader is referred to the recent survey [17]. If the illuminating energy is impulsive in time, the propagation may be modelled as an initial value problem for the wave equation. The problem of recovering the initial data of a solution of the wave equation from the value of the solution on the boundary of a domain is of mathematical interest in every dimension, but for the application to thermo-/photoacoustic tomography it would appear that the three dimensional case is the only one of interest, since sound propagation is not confined to a lower dimensional submanifold. However, there exist methods of measuring the generated wave field which do not rely on point measurements of the sort that would be generated by an (idealized) acoustic transducer. In particular, integrating line detectors, which have been studied in [3, 14], in effect compute the integral of the acoustic wave field along a specified line. In this paper, we work under the assumption that the speed of sound, $c$, is constant throughout the body, and since the x-ray transform in a given direction of a solution of the three dimensional wave equation is a solution of the two dimensional wave equation, the problem is transformed. If a circular array of line detectors is rotated around an axis orthogonal to the direction of the line detectors [7, 14], then for each fixed rotation angle the measurement provides the trace of the solution of the two dimensional wave equation on the circle corresponding to the array. The initial data of this two dimensional problem is the x-ray transform of the three dimensional initial data. If the inital data can be recovered in the disk bounded by the detector array and assuming that the projection of the object to be imaged lies in this disk, then the problem of recovering the three dimensional initial data is reduced to the inversion of the x-ray transform in each

plane orthogonal to the axis of rotation. One such two dimensional problem is illustrated in Figure 1,

To our knowledge, the first work to tackle the problem of recovering a function from its circular means with centers on a circle was [13], whose author was interested in ultrasound reflectivity tomography. He found an inversion method based on harmonic decomposition and for each harmonic, the inversion of a Hankel transform. This method has been the basis for most subsequent work on exact inversion of circular means. The inversion of the Hankel 


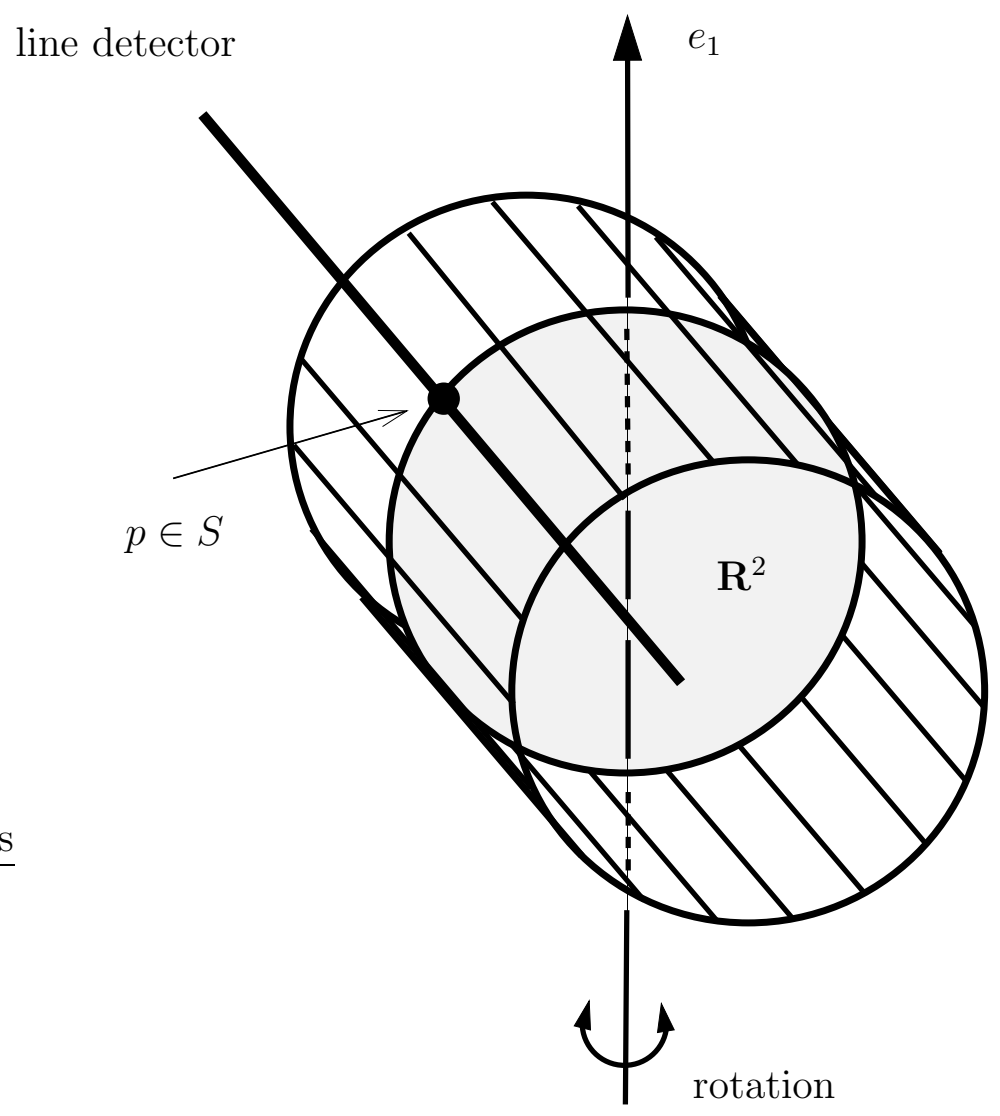

Figure 1: Principle of thermoacoustic tomography with integrating line detectors. A cylindrical array of line detectors records the acoustic field and is rotated around the axis $e_{1}$. For fixed rotation angle the array outputs the x-ray transform (projection along straight lines) of the solution of the wave equation restricted to lines passing through the boundary $S$ of the disk. The initial condition is given by the x-ray transform of the initially induced pressure restricted to lines orthogonal to the base of the cylinder.

transform involves a quotient of a Hankel transform of a harmonic component of the data and a Bessel function. That this quotient be well-defined turns out to be a condition on the range of the circular mean transform [2. See also [1] for range results on the spherical mean transform on functions supported in a ball in all dimensions, and [6] for range results for the wave trace map for functions supported in the ball in odd dimensions.

In the work of the first and third authors with Sarah Patch [5], several formulas were found to recover a smooth function $f$ with support in the closure $\bar{B}$ of the open ball $B \subseteq \mathbf{R}^{n}$ from the trace of the solution of the wave equation on the product $\partial B \times[0, \operatorname{diam}(B)]$ provided 
that the space dimension is odd. Specifically, if $u$ is the solution of the initial value problem

$$
\begin{gathered}
u_{t t}-\Delta u=0, \quad \text { in } \mathbf{R}^{n} \times[0, \infty) \\
u(., t=0)=f(.), \quad u_{t}(., t=0)=0,
\end{gathered}
$$

where $f$ is smooth and has support in the $\bar{B}$, then several formulas were found to recover $f$ from $u(p, t)$ for $p \in S:=\partial B$ and $t \in \mathbf{R}^{+}$.

The first and third authors tried, at that time, to extend the method to even dimensions, but did not see a way. Recently, the second author tried numerical experiments using a two dimensional analog of one of the inversion formulas and found that it gave excellent reconstructions. This prompted our re-examination of the problem. Among the results of this paper is a proof of the validity of this formula.

To describe our results, we introduce some notation. The spherical mean transform $\mathcal{M}$ is defined by

$$
(\mathcal{M} f)(x, r)=\frac{1}{\left|S^{n-1}\right|} \int_{S^{n-1}} f(x+r \theta) d S(\theta)
$$

for $f \in C^{\infty}\left(\mathbf{R}^{n}\right)$ and $(x, r) \in \mathbf{R}^{n} \times[0, \infty)$. In this expression, $\left|S^{n-1}\right|$ denotes the area of the unit sphere $S^{n-1}$ in $\mathbf{R}^{n}$ and $d S(\theta)$ denotes area measure on the sphere. In general, we write the area measure on a sphere of any radius as $d S$, except when $n=2$ when we write $d s$. We will denote the (partial) derivative of a function $q$ with respect to a variable $r$ by $\partial_{r} q$, except in a few formulas where the subscript notation $q_{r}$ is used. At several points we use $D_{r}$ to denote the operator

$$
\left(D_{r} u\right)(r):=\frac{\left(\partial_{r} u\right)(r)}{2 r}
$$

acting on smooth (even) functions $u$ with compact support. Moreover, $r$ will be used to denote the operator that multiplies a function $u(r)$ by $r$.

Our first set of results is a pair of inversion formulas for the spherical mean transform in even dimensions. We state and prove these first in dimension two; that is, for the circular mean transform.

Theorem 1. Let $D \subset \mathbf{R}^{2}$ be the disk of radius $R_{0}$ centered at the origin, let $S:=\partial D$ denote the boundary circle, and let $f \in C^{\infty}\left(\mathbf{R}^{2}\right)$ with supp $f \subset \bar{D}$. Then, for $x \in D$,

$$
f(x)=\frac{1}{2 \pi R_{0}} \Delta_{x} \int_{S} \int_{0}^{2 R_{0}} r(\mathcal{M} f)(p, r) \log \left|r^{2}-\right| x-\left.p\right|^{2} \mid d r d s(p)
$$

and

$$
f(x)=\frac{1}{2 \pi R_{0}} \int_{S} \int_{0}^{2 R_{0}}\left(\partial_{r} r \partial_{r} \mathcal{M} f\right)(p, r) \log \left|r^{2}-\right| x-\left.p\right|^{2} \mid d r d s(p)
$$


In Theorem 1, $\partial_{r} r \partial_{r} \mathcal{M} f$ denotes the composition of $\partial_{r}, r, \partial_{r}$ and $\mathcal{M}$ applied to $f$. The same convention will be used throughout the article to denote the composition of any operators.

While $\mathcal{M} f$ has a natural extension to the negative reals as an even function, we instead take the odd extension in the second variable. Then formula (5) has the following corollary:

Corollary 1. With the same hypotheses as in Theorem 1 , and $\mathcal{M} f$ extended as an odd function in the second variable $r, f$ can be recovered for $x \in D$ by

$$
f(x)=\frac{1}{2 \pi R_{0}} \int_{S} \int_{-2 R_{0}}^{2 R_{0}} \frac{\left(r \partial_{r} \mathcal{M} f\right)(p, r)}{|x-p|-r} d r d s(p),
$$

and

$$
f(x)=\frac{1}{2 \pi R_{0}} \int_{S}|x-p| \int_{-2 R_{0}}^{2 R_{0}} \frac{\left(\partial_{r} \mathcal{M} f\right)(p, r)}{|x-p|-r} d r d s(p),
$$

where the inner integrals are taken in the principal value sense.

These forms are very close to the standard inversion formula for the Radon transform in the plane [12, Eq. (2.5)].

In higher even dimensions we prove a similar pair of results.

Theorem 2. Let $B \subset \mathbf{R}^{n}, n>2$ even, be the ball of radius $R_{0}$ centered at the origin, let $S:=\partial B$ be the boundary of the ball, set

$$
c_{n}=(-1)^{(n-2) / 2} 2((n-2) / 2) ! \pi^{n / 2}=(-1)^{(n-2) / 2}[((n-2) / 2) !]^{2}\left|S^{n-1}\right|,
$$

and let $f \in C^{\infty}\left(\mathbf{R}^{n}\right)$ have support in $\bar{B}$. Then, for $x \in B$,

$$
\begin{aligned}
& f(x)=\frac{1}{c_{n} R_{0}} \Delta_{x} \int_{S} \int_{0}^{2 R_{0}} \log \left|r^{2}-\right| x-\left.p\right|^{2} \mid\left(r D_{r}^{n-2} r^{n-2} \mathcal{M} f\right)(p, r) d r d S(p), \\
& f(x)=\frac{2}{c_{n} R_{0}} \int_{S} \int_{0}^{2 R_{0}} \log \left|r^{2}-\right| x-\left.p\right|^{2} \mid\left(r D_{r}^{n-1} r^{n-1} \partial_{r} \mathcal{M} f\right)(p, r) d r d S(p) .
\end{aligned}
$$

Recently, Kunyansky [1] has also established inversion formulas of the filtered backprojection type for the spherical mean transform. His method and results appear to be very different than ours.

For some results, it will be more convenient to use the wave equation (1) with initial condition

$$
u(., t=0)=0, \quad u_{t}(., t=0)=f(.)
$$


It is obvious that the solution of (11) with initial values (2) is the time derivative of the solution of (11) with initial values (10). We denote by $\mathcal{P}$ the operator which takes smooth initial data with support in $\bar{B}$ to the solution of (1), (10) restricted to $S \times[0, \infty)$ and by $\mathcal{W}$ the operator taking $f$ to the solution of (11), (2) restricted to $S \times[0, \infty)$. These operators are simply related by $\mathcal{W}=\partial_{t} \mathcal{P}$. An explicit representation for $\mathcal{P}$ comes from the well-known formula [4]

$$
u(p, t)=\frac{1}{(n-2) !} \partial_{t}^{n-2} \int_{0}^{t} r\left(t^{2}-r^{2}\right)^{(n-3) / 2}(\mathcal{M} f)(p, r) d r .
$$

giving the solution of the initial value problem (1), (10), in dimension $n \geq 2$. We denote by $\mathcal{P}^{*}$ and $\mathcal{W}^{*}=-\mathcal{P}^{*} \partial_{t}$ the formal $L^{2}$ adjoints of $\mathcal{P}$ and $\mathcal{W}$ mapping from smooth functions $u \in C^{\infty}(S \times[0, \infty))$ with sufficient decay in the second variable. An explicit expression for $\mathcal{P}^{*} u$ will be given in Section 3 ,

We have two types of inversion results for the wave equation. The first type is based on the inversion results for the spherical mean transform, since the spherical mean transform itself can be recovered from the solution of the wave equation by solving an Abel type equation. In dimension two, this approach yields the following result.

Theorem 3. Let $D \subset \mathbf{R}^{2}$ be the open disc with radius $R_{0}$ and let $S:=\partial D$ denote the boundary circle. Then there exists a kernel function $K:\left[0,2 R_{0}\right]^{2} \rightarrow \mathbf{R}$ such that for any $f \in C^{\infty}\left(\mathbf{R}^{2}\right)$ with support in $\bar{D}$ and any $x \in D$

$$
f(x)=\frac{1}{R_{0} \pi^{2}} \Delta_{x} \int_{S} \int_{0}^{2 R_{0}}(\mathcal{W} f)(p, t) K(t,|x-p|) d t d s(p) .
$$

An analytic expression for $K$ will be given in Section 3 .

Theorem 3 provides inversion formulas of the filtered back-projection type for reconstruction of $f$ from $(\mathcal{W} f)(p, t)=\left(\partial_{t} \mathcal{P} f\right)(p, t)$ using only data with $t \in\left[0,2 R_{0}\right]$, despite the unbounded support of $\mathcal{W} f$ and $\mathcal{P} f$ in $t$.

The second type of inversion results holds in all even dimensions and takes the following form.

Theorem 4. Let $f$ be smooth and supported in closure of the ball $B$ of radius $R_{0}$ in $\mathbf{R}^{2 m}$, and let $\mathcal{P} f$ and $\mathcal{W} f$ be as above. Then for $x \in B$

$$
\begin{aligned}
f(x) & =-\frac{2}{R_{0}}\left(\mathcal{P}^{*} t \partial_{t}^{2} \mathcal{P} f\right)(x) \\
f(x) & =\frac{2}{R_{0}}\left(\mathcal{W}^{*} t \mathcal{W} f\right)(x)=-\frac{2}{R_{0}}\left(\mathcal{P}^{*} \partial_{t} t \partial_{t} \mathcal{P} f\right)(x) .
\end{aligned}
$$


We will prove (13) in dimension $n=2 m=2$ directly. The higher dimensional case of (13), and (14) in all dimensions, are consequences of the following trace identities, relating

the $L^{2}$ inner product of the initial data to the weighted $L^{2}$ inner product of the traces of the solutions of the wave equation.

Theorem 5. Let $f, g$ be smooth and supported in the ball $B$ of radius $R_{0}$, in $\mathbf{R}^{2 m}$ with $m \geq 1$, let $S:=\partial B$, and let $u$ (resp. $v$ ) be the solution of the initial value problem (1), (10) with initial value $f$ (resp. $g$ ). Then

$$
\begin{aligned}
& \int_{B} f(x) g(x) d x=-\frac{2}{R_{0}} \int_{S} \int_{0}^{\infty} t u_{t t}(p, t) v(p, t) d t d S(p), \\
& \int_{B} f(x) g(x) d x=\frac{2}{R_{0}} \int_{S} \int_{0}^{\infty} t u_{t}(p, t) v_{t}(p, t) d t d S(p) .
\end{aligned}
$$

In the proof of this theorem, (15) for $n=2$ follows from (13) for $n=2$, while (15) in higher even dimensions is derived from the $n=2$ case; (16) is a consequence of (15) in all dimensions. We remark that these identities were already proved in [5] for odd dimensions, and so they hold for all dimensions.

Section 2 is devoted to the proof of the inversion formulas for the spherical mean transform, that is, Theorems 1, 2, and Corollary 1. Section 3 treats the wave equation and contains the proofs of Theorems 3, 4, and 5. This is followed by a section reporting on the implementation of the various reconstruction formulas of the preceding sections and results of numerical tests, in dimension two.

\section{Spherical Means}

In this section we prove the Theorems related to the inversion from spherical means and Corollary 1. We begin by establishing an elementary integral identity, which is the key to the results in this paper.

Proposition 2.1. Let $D \subseteq \mathbf{R}^{2}$ be the disk of radius $R_{0}$, and let $S=\partial D$ be the boundary circle. Then for $x, y \in D$ with $x \neq y$,

$$
\int_{S} \log || x-\left.p\right|^{2}-|y-p|^{2}\left|d s(p)=2 \pi R_{0} \log \right| x-y \mid+2 \pi R_{0} \log R_{0} .
$$

Proof. Let $x \neq y$ both lie in $D$ and let $I$ denote the integral on the left on (17). Expanding 
the argument of the logarithm as

$$
|| x-\left.p\right|^{2}-|y-p|^{2}\left|=2 R_{0}\right| x-y||\left(\frac{x+y}{2 R_{0}}-\frac{p}{R_{0}}\right) \cdot \frac{x-y}{|x-y|} \mid,
$$

setting $e:=\frac{x-y}{|x-y|}$, and writing $p=R_{0} \theta$ for $\theta \in S^{1}$, we have

$$
I=2 \pi R_{0} \log \left(2 R_{0}|x-y|\right)+R_{0} \int_{S^{1}} \log |e \cdot \theta-a| d \theta
$$

where

$$
a=\frac{x+y}{2 R_{0}} \cdot e=\frac{|x|^{2}-|y|^{2}}{2 R_{0}|x-y|}
$$

We note that $|a|<1$.

Using the parameterization $\theta=\cos (\phi) e+\sin (\phi) e^{\perp}$, the integral term on the right of (18) has the form

$$
R_{0} \int_{0}^{2 \pi} \log |\cos \phi-a| d \phi
$$

Writing $a=\cos \alpha$ and using the sum to product trigonometric identity $\cos \phi-\cos \alpha=$ $-2 \sin ((\phi+\alpha) / 2) \sin ((\phi-\alpha) / 2)$, this is equal to

$$
R_{0} \int_{0}^{2 \pi}(\log 2+\log |\sin ((\phi+\alpha) / 2)|+\log |\sin ((\phi-\alpha) / 2)|) d \phi .
$$

By periodicity, and two linear changes of variable, this reduces to

$$
R_{0} \int_{0}^{2 \pi}(\log 2+2 \log |\sin (\phi / 2)|) d \phi=2 R_{0} \pi \log 2+4 R_{0} \int_{0}^{\pi} \log \sin u d u
$$

which is independent of $\alpha$, and hence of $x$ and $y$. The latter integral in can be found in tables, and is equal to $-R_{0} \pi \log 2$, so the sum is $-2 \pi R_{0} \log 2$. Substituting in (18) gives the desired result.

Proposition 2.1 is already enough to establish Theorem 1 .

Proof of Theorem [1. Let $f \in C^{\infty}\left(\mathbf{R}^{2}\right)$ be supported in $\bar{D}$ and let $p$ be any point in $S=\partial B$. Using the definition of $\mathcal{M} f$ and Fubini's theorem, we have that

$$
\int_{0}^{2 R_{0}}(r \mathcal{M} f)(p, r) q(r) d r=\frac{1}{2 \pi} \int_{\mathbf{R}^{2}} f(p+z) q(|z|) d z
$$


for any measurable function $q$ provided that the product of functions on the right is absolutely integrable. Applying this with $q(r)=\log \left|r^{2}-\right| x-\left.p\right|^{2} \mid$ and making the change of variables $y=p+z$ gives

$$
\begin{aligned}
\int_{S} \int_{0}^{2 R_{0}}(r \mathcal{M})(f)(p, r) \log \left|r^{2}-\right| x-\left.p\right|^{2} \mid & d r d s(p) \\
& =\frac{1}{2 \pi} \int_{S} \int_{\mathbf{R}^{2}} f(y) \log || y-\left.p\right|^{2}-|x-p|^{2} \mid d y d s(p) .
\end{aligned}
$$

Fubini's theorem again justifies the change of order of integration in the iterated integral on the right hand side, and so

$$
\frac{1}{2 \pi} \int_{\mathbf{R}^{2}} f(y) \int_{S} \log || y-\left.p\right|^{2}-|x-p|^{2} \mid d s(p) d y=\frac{2 \pi R_{0}}{2 \pi} \int_{\mathbf{R}^{2}} f(y)\left(\log |x-y|+\log R_{0}\right) d y
$$

upon application of (17). Recalling that for any constant $c, 1 /(2 \pi) \log |x-y|+c$ is a fundamental solution of the Laplacian in $\mathbf{R}^{2}$, we have

$$
f(x)=\frac{1}{2 \pi R_{0}} \Delta_{x} \int_{S} \int_{0}^{2 R_{0}}(r \mathcal{M} f)(p, r) \log \left|r^{2}-\right| x-\left.p\right|^{2} \mid d r d s(p)
$$

which proves (4).

The second formula, (5), has a similar proof. In this case, we use that the spherical means satisfy the Euler-Poisson-Darboux equation [4]

$$
\left(\partial_{r}^{2} \mathcal{M} f\right)(x, r)+\frac{1}{r}\left(\partial_{r} \mathcal{M} f\right)(x, r)=(\Delta \mathcal{M} f)(x, r)=(\mathcal{M} \Delta f)(x, r) .
$$

The left hand side of the Darboux equation may be written as $(1 / r)\left(\partial_{r} r \partial_{r} \mathcal{M} f\right)(x, r)$, so the expression on the right of (5) may be rewritten as

$$
\frac{1}{2 \pi R_{0}} \int_{S} \int_{0}^{2 R_{0}}(r \mathcal{M} \Delta f)(p, r) \log \left|r^{2}-\right| x-\left.p\right|^{2} \mid d r d s(p) .
$$

Again applying (19), now with the function $q(r)=r \log \left|r^{2}-\right| x-\left.p\right|^{2} \mid$ and $\Delta f$ instead of $f$, interchanging the order of integration and using (17) shows that the expression (20) is equal to

$$
\frac{1}{2 \pi} \int_{\mathbf{R}^{2}} \Delta_{y} f(y)\left(\log |x-y|+\log R_{0}\right) d y=f(x),
$$

since no boundary terms arise in view of the support hypothesis on $f$.

Proof of Corollary 1. Let $x \in D$, and let

$$
U(p, x):=\int_{0}^{2 R_{0}}\left(\partial_{r} r \partial_{r} \mathcal{M} f\right)(p, r) \log \left|r^{2}-\right| x-\left.p\right|^{2} \mid d r
$$


denote the inner integral in (5). Taking the support of $f$ into account, writing the logarithm as

$$
\log \left|r^{2}-\right| x-\left.p\right|^{2}|=\log | r-|x-p||+\log | r+|x-p| \mid,
$$

and integrating (5) by parts leads to

$$
U(p, x)=-P . V . \int_{0}^{\infty} \frac{\left(r \partial_{r} \mathcal{M} f\right)(p, r)}{r-|x-p|} d r-\int_{0}^{\infty} \frac{\left(r \partial_{r} \mathcal{M} f\right)(p, r)}{r+|x-p|} d r .
$$

Here we have used that the distributional derivative of $\log |r|$ is $P . V . \frac{1}{r}$ as well as an ordinary integration by parts. Therefore (5) implies

$$
\begin{aligned}
f(x) & =\frac{1}{2 \pi R_{0}} \int_{S} U(p, r) d s(p) \\
& =\frac{-1}{2 \pi R_{0}} \int_{S} \int_{0}^{2 R_{0}} \frac{\left(r \partial_{r} \mathcal{M} f\right)(p, r)}{r-|x-p|} d r d s(p)+\frac{-1}{2 \pi R_{0}} \int_{S} \int_{0}^{2 R_{0}} \frac{\left(r \partial_{r} \mathcal{M} f\right)(p, r)}{r+|x-p|} d r d s(p),
\end{aligned}
$$

where the inner integral of the first term on the right is taken in the principal value sense. The odd extension of $\mathcal{M} f, \mathcal{M} f(p,-r):=-\mathcal{M} f(p, r)$, is smooth on $\mathbf{R}$ since $\mathcal{M} f$ vanishes to infinite order at $r=0$ by the support hypothesis on $f$ and $\left(r \partial_{r} \mathcal{M} f\right)(p, r)$ is an odd function in $r$. Substituting $r=-r$ in the second integral in (21) gives

$$
f(x)=\frac{-1}{2 \pi R_{0}} \int_{S} \int_{0}^{2 R_{0}} \frac{\left(r \partial_{r} \mathcal{M} f\right)(p, r)}{r-|x-p|} d r d s(p)+\frac{-1}{2 \pi R_{0}} \int_{S} \int_{-2 R_{0}}^{0} \frac{\left(r \partial_{r} \mathcal{M} f\right)(p, r)}{r-|x-p|} d r d s(p)
$$

and hence

$$
f(x)=\frac{1}{2 \pi R_{0}} \int_{S} \int_{-2 R_{0}}^{2 R_{0}} \frac{\left(r \partial_{r} \mathcal{M} f\right)(p, r)}{|x-p|-r} d r d s(p) .
$$

This is (6). To prove (7), it suffices to write

$$
\frac{r}{|x-p|-r}=-1+\frac{|x-p|}{|x-p|-r}
$$

in (66) and to note that $\int_{-2 R_{0}}^{2 R_{0}}\left(\partial_{r} \mathcal{M} f\right)(p, r) d r=0$, by the support hypothesis on $f$.

\subsection{Proof of Theorem 2}

We have found several proofs of Theorem 2, the extension of Theorem 1 to higher even dimensions. The one we present is based on reduction of the higher dimensional problem to the two dimensional case already established. Another, which is not presented in this article, is based on an extension of (17) to higher dimensions. 
We first observe that by a dilation, we may reduce the problem to the case when $f$ is supported in the unit ball. Tracing through the formulas (8) and (9) it is routine to verify that scaling from the unit ball to the ball of radius $R_{0}$ introduces a factor of $R_{0}$. To simplify notation, we shall now suppose that $f$ is supported in the unit ball $B$. Let $Q$ and $N$ denote the operators

$$
\begin{aligned}
& (Q f)(x)=\Delta_{x} \int_{S} \int_{0}^{2}\left(r D_{r}^{n-2} r^{n-2} \mathcal{M} f\right)(p, r) \log \left|r^{2}-\right| x-\left.p\right|^{2} \mid d r d S(p), \\
& (N f)(x)=\int_{S} \int_{0}^{2}\left(r D_{r}^{n-1} r^{n-1} \partial_{r} \mathcal{M} f\right)(p, r) \log \left|r^{2}-\right| x-\left.p\right|^{2} \mid d r d S(p),
\end{aligned}
$$

that map $f \in C^{\infty}\left(\mathbf{R}^{n}\right)$ supported in $\bar{B}$ to constant multiples of the the right hand sides of (8) and (9). Moreover $\langle f, g\rangle$ denotes the $L^{2}$ product of two functions supported in $\bar{B}$. To establish $Q f=c_{n} f$ and $N f=\left(c_{n} / 2\right) f$ we will use the following auxiliary results.

Proposition 2.2. Let $f, g$ be smooth and supported in $\bar{B}$. Then

$$
\int_{\mathbf{R}^{n}}(Q f)(x) g(x) d x=\langle Q f, g\rangle=2\langle f, N g\rangle=2 \int_{\mathbf{R}^{n}} f(x)(N g)(x) d x .
$$

Proof. Let $F=\mathcal{M} f$ and $G=\mathcal{M g}$. Using the self-adjointness of $\Delta$, applying Fubini's theorem and an $n$-dimensional analogue of (19), we obtain

$$
\begin{aligned}
\langle Q f, g\rangle & =\int_{B}\left(\int_{S} \int_{0}^{2}\left(r D_{r}^{n-2} r^{n-2} F\right)(p, r) \log \left|r^{2}-\right| x-\left.p\right|^{2} \mid d r d S(p)\right)\left(\Delta_{x} g\right)(x) d x \\
& =\left|S^{n-1}\right| \int_{S} \int_{0}^{2}\left(\int_{0}^{2}\left(r D_{r}^{n-2} r^{n-2} F\right)(p, r) \log \left|r^{2}-\bar{r}^{2}\right|\left(\mathcal{M} \Delta_{x} g\right)(p, \bar{r}) \bar{r}^{n-1} d \bar{r}\right) d r d S(p) \\
& =\left|S^{n-1}\right| \int_{S} \int_{0}^{2}\left(\int_{0}^{2}\left(r D_{r}^{n-2} r^{n-2} F\right)(p, r) \log \left|r^{2}-\bar{r}^{2}\right| d r\right)\left(\mathcal{M} \Delta_{x} g\right)(p, \bar{r}) \bar{r}^{n-1} d \bar{r} d S(p) \\
& =\left|S^{n-1}\right| \int_{S} \int_{0}^{2}\left(\int_{0}^{2}\left(r D_{r}^{n-2} r^{n-2} F\right)(p, r) \log \left|r^{2}-\bar{r}^{2}\right| d r\right) \partial_{\bar{r}} \bar{r}^{n-1} \partial_{\bar{r}} G(p, \bar{r}) d \bar{r} d S(p) .
\end{aligned}
$$

To justify the last equation it is used that $G$ satisfies the Euler-Poisson-Darboux equation and the identity $\bar{r}^{n-1}\left(\partial_{\bar{r}}^{2}+\frac{n-1}{\bar{r}} \partial_{\bar{r}}\right)=\partial_{\bar{r}}\left(\bar{r}^{n-1} \partial_{\bar{r}}\right)$. Applying the identities $\left(D_{r}^{n-2}\right)^{*} r \log \left|r^{2}-\bar{r}^{2}\right|=$ $(-1)^{n-2} r D_{r}^{n-2} \log \left|r^{2}-\bar{r}^{2}\right|=r D_{\bar{r}}^{n-2} \log \left|r^{2}-\bar{r}^{2}\right|$ in two stages to the last expression, this becomes

$$
\begin{aligned}
\left|S^{n-1}\right| & \int_{S} \int_{0}^{2}\left(\int_{0}^{2} r^{n-1} F(p, r) D_{\bar{r}}^{n-2} \log \left|r^{2}-\bar{r}^{2}\right| d r\right)\left(\partial_{\bar{r}} \bar{r}^{n-1} \partial_{\bar{r}} G(p, \bar{r})\right) d \bar{r} d S(p) \\
& =\int_{S} \int_{0}^{2}\left(\int_{B} f(y) D_{\bar{r}}^{n-2} \log || y-\left.p\right|^{2}-\bar{r}^{2} \mid d y\right)\left(\partial_{\bar{r}} \bar{r}^{n-1} \partial_{\bar{r}} G(p, \bar{r})\right) d \bar{r} d S(p) \\
& =\int_{B}\left(\int_{S} \int_{0}^{2} \log || y-\left.p\right|^{2}-\bar{r}^{2} \mid\left(\left(D_{\bar{r}}^{*}\right)^{n-2} \partial_{\bar{r}} \bar{r}^{n-1} \partial_{\bar{r}} G\right)(p, \bar{r}) d \bar{r} d S(p)\right) f(y) d y
\end{aligned}
$$


after applying Fubini's theorem. This is finally seen to be equal to $\langle f, 2 N g\rangle$ since $\left(D_{\bar{r}}^{n-2}\right)^{*} \partial_{\bar{r}}=$ $2 \bar{r}(-1)^{n-2} D_{\bar{r}}^{n-1}$.

We now look at the spherical means of products

$$
f(x)=\rho^{k} \alpha(\rho) \Phi(\theta),
$$

where $x=\rho \theta$ with $\rho \geq 0, \theta \in S^{n-1}, \Phi$ is a spherical harmonic of degree $k$, and $\alpha: \mathbf{R} \rightarrow \mathbf{R}$ is an even smooth function supported in $[-1,1]$. Let $F:=\mathcal{M} f$ be extended to an even function in the second component and let $\nu=n+2 k$. Then $F$ satisfies the initial value problem (IVP) for the Euler-Poisson-Darboux equation

$$
\begin{array}{ll}
\left(\partial_{r}^{2} F+\frac{n-1}{r} \partial_{r} F\right)(x, r)=\Delta_{x} F(x, r), & (x, r) \in \mathbf{R}^{n} \times \mathbf{R} \\
F(x, 0)=\alpha(\rho) \rho^{k} \Phi(\theta), & \partial_{r} F(x, 0)=0,
\end{array}
$$

and, conversely, any solution of (27), (28), is the spherical mean of the initial values. The unique solution of (27), (28) has the form $F(x, r)=\rho^{k} A(\rho, r) \Phi(\theta)$ where $A(\rho, r)$ is the solution of the IVP

$$
\begin{array}{cc}
\left(L_{n} A\right)(\rho, r)=\left(\partial_{\rho}^{2} A+\frac{\nu-1}{\rho} \partial_{\rho} A\right)(\rho, r), & (\rho, r) \in \mathbf{R}^{2}, \\
A(\rho, 0)=\alpha(\rho), \quad \partial_{\rho} A(\rho, 0)=0, & \rho \in \mathbf{R} .
\end{array}
$$

Here $\left(L_{n} A\right)(\rho, r):=\left(\partial_{r}^{2} A+\frac{n-1}{r} \partial_{r} A\right)(\rho, r)$.

We recall that the operator $D_{r}$ satisfies $L_{n} D_{r}=D_{r} L_{n-2}$ and for any $\mu \in \mathbf{N}$

$$
\left(\partial_{r}^{2}+\frac{1-\mu}{r} \partial_{r}\right)\left(r^{\mu} w\right)=r^{\mu}\left(\partial_{r}^{2}+\frac{1+\mu}{r} \partial_{r}\right) w
$$

that is $L_{2-\mu} r^{\mu}=r^{\mu} L_{\mu+2}$. So

$$
\left(L_{2-\mu+2 \sigma} D_{r}^{\sigma} r^{\mu} w\right)(r)=\left(D_{r}^{\sigma} L_{2-\mu} r^{\mu} w\right)(r)=\left(D_{r}^{\sigma} r^{\mu} L_{\mu+2} w\right)(r) .
$$

If we set $\mu=n-2$ and $\sigma=(n-2) / 2$ in (31), then $\mu+2=n$ and $2-\mu+2 \sigma=2$. Therefore

$$
\left(L_{2} D_{r}^{(n-2) / 2} r^{n-2} w\right)(r)=\left(D_{r}^{(n-2) / 2} r^{n-2} L_{n} w\right)(r) .
$$

Now we set

$$
H(\rho, r):=\frac{1}{((n-2) / 2) !}\left(D_{r}^{(n-2) / 2} r^{n-2} A\right)(\rho, r)
$$


Since $A(\rho, r)$ is even in $r$ and $D_{r}$ corresponds to differentiation with respect to $r^{2}, H(\rho, r)$ is even in $r$. Moreover, by (301), $H(\rho, 0)=\frac{1}{((n-2) / 2) !} A(\rho, 0)\left(D_{r}^{(n-2) / 2} r^{n-2}\right)=\alpha(\rho)$, and therefore from (29) and (32) it follows that $H$ is the solution of the IVP

$$
\begin{array}{cl}
\left(\partial_{r}^{2} H+\frac{1}{r} \partial_{r} H\right)(\rho, r)=\left(\partial_{\rho}^{2} H+\frac{\nu-1}{\rho} \partial_{\rho} H\right)(\rho, r), & (\rho, r) \in \mathbf{R}^{2}, \\
H(\rho, 0)=\alpha(\rho), \quad \partial_{r} H(\rho, 0)=0, \quad \rho \in \mathbf{R},
\end{array}
$$

Proposition 2.3. Let $A_{i}(\rho, r), i=1,2$ solve (29) with $n=2$, subject to initial conditions $A_{i}(\rho, 0)=\alpha_{i}(\rho), \partial_{r} A_{i}(\rho, 0)=0$, where $\alpha_{i}$ are smooth even functions with support in $[-1,1]$ and $\nu \geq 2$ is even. Then

$$
\int_{0}^{1} \rho^{\nu-1} \alpha_{1}(\rho) \alpha_{2}(\rho) d \rho=-\int_{0}^{2} \int_{0}^{2} r A_{1}(1, r) \partial_{\bar{r}} \log \left|r^{2}-\bar{r}^{2}\right| \bar{r}\left(\partial_{\bar{r}} A_{2}\right)(1, \bar{r}) d \bar{r} d r .
$$

Proof. Let $k=(\nu-2) / 2$ and let $\Phi(\theta)$ be a nontrivial real circular harmonic of degree $k$. Then $F_{i}(x, r):=A_{i}(\rho, r) \rho^{k} \Phi(\theta)$ satisfies (27), (28) for $n=2$, and so is the circular mean of its initial value, $f_{i}(x)=\alpha_{i}(\rho) \rho^{k} \Phi(\theta)$. By (4), $f_{1}=\frac{1}{2 \pi} Q f_{1}$, and using (25) gives

$$
\begin{aligned}
\left\langle f_{1}, f_{2}\right\rangle & =\frac{1}{2 \pi}\left\langle Q f_{1}, f_{2}\right\rangle \\
& =\int_{S} \int_{0}^{2} \int_{0}^{2} r F_{1}(p, r) \log \left|r^{2}-\bar{r}^{2}\right|\left(\partial_{\bar{r}} \bar{r} \partial_{\bar{r}} F_{2}\right)(p, \bar{r}) d r d \bar{r} d s(p) .
\end{aligned}
$$

Taking account the form of $F_{i}$ and that $\rho=1$ on $S$, this may be rewritten as

$$
\left\langle f_{1}, f_{2}\right\rangle=\int_{S} \Phi^{2}(p) d s(p) \int_{0}^{2} \int_{0}^{2} r A_{1}(1, r) \log \left|r^{2}-\bar{r}^{2}\right|\left(\partial_{\bar{r}} \bar{r} \partial_{\bar{r}} A_{2}\right)(1, \bar{r}) d r d \bar{r} .
$$

Appealing to the form of $f_{i}=F(x, 0)$,

$$
\begin{aligned}
\left\langle f_{1}, f_{2}\right\rangle & =\int_{0}^{1} \rho\left(\rho^{k} \alpha_{1}\right)\left(\rho^{k} \alpha_{2}\right) d \rho \int_{S} \Phi^{2}(p) d s(p) \\
& =\int_{0}^{1} \rho^{\nu-1} \alpha_{1}(\rho) \alpha_{2}(\rho) d \rho \int_{S} \Phi^{2}(p) d s(p) .
\end{aligned}
$$

Since $\int_{S} \Phi^{2}(p) d s(p) \neq 0$, a comparison of (37) and (38) and an integration by parts on the right side of (37) establishes (36) which completes the proof.

Proof of Theorem 2. Let $\left\{\Phi_{j}\right\}$ be an orthonormal basis for the spherical harmonics on $S^{n-1}$, and consider $f_{i}, i=1,2$ of the form (26) with $\alpha=\alpha_{i}$ and $\Phi=\Phi_{j_{i}}$ of possibly different degrees. Let $F_{i}$ be the even extensions of $\mathcal{M} f_{i}$ as above. Then by orthogonality, $\left\langle f_{1}, f_{2}\right\rangle=0$ unless $j_{1}=j_{2}$, in which case

$$
\left\langle f_{1}, f_{2}\right\rangle=\int_{0}^{1} \rho^{\nu-1} \alpha_{1}(\rho) \alpha_{2}(\rho) d \rho
$$


with $\nu=n+2 k$, where $k$ is the degree of $\Phi_{j_{1}}$. Evaluating $\left\langle Q f_{1}, f_{2}\right\rangle$ by (25), and using that $F_{i}=\rho^{k_{i}} A_{i}(\rho, r) \Phi_{j_{i}}$, we see that it is also zero unless $j_{1}=j_{2}$. In this case we have

$$
\begin{aligned}
& \left\langle Q f_{1}, f_{2}\right\rangle=\left|S^{n-1}\right| \int_{0}^{2} \int_{0}^{2}\left(r D_{r}^{n-2} r^{n-2} A_{1}\right)(1, r) \log \left|r^{2}-\bar{r}^{2}\right|\left(\partial_{\bar{r}} \bar{r}^{n-1} \partial_{\bar{r}} A_{2}\right)(1, \bar{r}) d r d \bar{r} \\
& \quad=\left|S^{n-1}\right| \int_{0}^{2} \int_{0}^{2}\left(D_{r}^{\frac{n-2}{2}} r^{n-2} A_{1}\right)(1, r)\left(D_{r}^{*}\right)^{\frac{n-2}{2}}\left(r \log \left|r^{2}-\bar{r}^{2}\right|\right)\left(\partial_{\bar{r}} \bar{r}^{n-1} \partial_{\bar{r}} A_{2}\right)(1, \bar{r}) d r d \bar{r} \\
& =\left|S^{n-1}\right| \int_{0}^{2} \int_{0}^{2} r\left(D_{r}^{\frac{n-2}{2}} r^{n-2} A_{1}\right)(1, r) D_{\bar{r}}^{\frac{n-2}{2}} \log \left|r^{2}-\bar{r}^{2}\right|\left(\partial_{\bar{r}} \bar{r}^{n-1} \partial_{\bar{r}} A_{2}\right)(1, \bar{r}) d r d \bar{r}
\end{aligned}
$$

since $\left(D_{r}^{(n-2) / 2}\right)^{*} r \log \left|r^{2}-\bar{r}^{2}\right|=(-1)^{(n-2) / 2} r D_{r}^{n-2} \log \left|r^{2}-\bar{r}^{2}\right|=r D_{\bar{r}}^{(n-2) / 2} \log \left|r^{2}-\bar{r}^{2}\right|$. Applying the adjoint (distributional derivative) again in (40),

$$
\begin{aligned}
& \left\langle Q f_{1}, f_{2}\right\rangle=\left|S^{n-1}\right| \int_{0}^{2} \int_{0}^{2} r\left(D_{r}^{\frac{n-2}{2}} r^{n-2} A_{1}\right)(1, r) \log \left|r^{2}-\bar{r}^{2}\right|\left(D_{\bar{r}}^{\frac{n-2}{2}}\right)^{*}\left(\partial_{\bar{r}} \bar{r}^{n-1} \partial_{\bar{r}} A_{2}\right)(1, \bar{r}) d r d \bar{r} \\
& =\left|S^{n-1}\right| \int_{0}^{2} \int_{0}^{2} r\left(D_{r}^{\frac{n-2}{2}} r^{n-2} A_{1}\right)(1, r) \log \left|r^{2}-\bar{r}^{2}\right|(-1)^{\frac{n-2}{2}}\left(\partial_{\bar{r}} D_{\bar{r}}^{\frac{n-2}{2}} \bar{r}^{n-1} \partial_{\bar{r}} A_{2}\right)(1, \bar{r}) d r d \bar{r} \\
& =\left|S^{n-1}\right|(-1)^{n / 2} \int_{0}^{2} \int_{0}^{2} r\left(D_{r}^{\frac{n-2}{2}} r^{n-2} A_{1}\right)(1, r) \partial_{\bar{r}} \log \left|r^{2}-\bar{r}^{2}\right|\left(D_{\bar{r}}^{\frac{n-2}{2}} \bar{r}^{n-1} \partial_{\bar{r}} A_{2}\right)(1, \bar{r}) d r d \bar{r} .
\end{aligned}
$$

We now use the following identity, which is readily proved by induction,

$$
D_{\bar{r}}^{(n-2) / 2} \bar{r}^{n-1} \partial_{\bar{r}} q=\bar{r} \partial_{\bar{r}} D_{\bar{r}}^{(n-2) / 2} \bar{r}^{n-2} q
$$

taking $q=A_{2}$, and observe that defining $H_{i}$ by (33) with $A=A_{i}$, it then holds that

$$
\left\langle Q f_{1}, f_{2}\right\rangle=\left|S^{n-1}\right|(-1)^{n / 2}[((n-2) / 2) !]^{2} \int_{0}^{2} \int_{0}^{2} r H_{1}(1, r) \partial_{\bar{r}} \log \left|r^{2}-\bar{r}^{2}\right| \bar{r}\left(\partial_{\bar{r}} H_{2}\right)(1, \bar{r}) d r d \bar{r} .
$$

By (34) and (35) the $H_{i}$ satisfy the hypotheses of Proposition 2.3 with initial data $\alpha_{i}$, so by (36) the expression on the right is equal to

$$
(-1)^{(n-2) / 2}\left|S^{n-1}\right|[((n-2) / 2) !]^{2} \int_{0}^{1} \rho^{\nu-1} \alpha_{1}(\rho) \alpha_{2}(\rho) d \rho .
$$

Thus we have proved that for $f_{i}$ of the form above

$$
\left\langle Q f_{1}, f_{2}\right\rangle=(-1)^{(n-2) / 2}\left|S^{n-1}\right|[((n-2) / 2) !]^{2}\left\langle f_{1}, f_{2}\right\rangle .
$$

We note that the constant on the right is $c_{n}$ of Theorem 2. By linearity and orthogonality of spherical harmonics, this still holds when either $f_{1}$ or $f_{2}$ is replaced by a finite linear combination of such functions. The set of finite linear combinations of functions of form (26) is dense in $L^{2}$, and so we have $Q f=c_{n} f$ in $L^{2}$ when $f$ is a finite linear combination 
of functions of the form (26). Now, let $g$ be smooth with with support in the unit ball. Applying Proposition 2.2, it follows that

$$
\langle f, N g\rangle=(1 / 2)\langle Q f, g\rangle=\left(c_{n} / 2\right)\langle f, g\rangle .
$$

for all $f$ as above. Since (42) holds for a dense subset of functions $f$ in $L^{2}(B)$, it implies that $N g=\left(c_{n} / 2\right) g$ almost everywhere in $B$. However, $N g$ is easily seen to be a continuous function, and so $N g=\left(c_{n} / 2\right) g$ holds pointwise in $B$, which is (9). But if $N$ is a multiple of the identity, then so $Q$, and the proof is complete.

\section{The Wave Equation}

We begin the analysis of recovery of initial data from the trace of the solution of the wave equation on the lateral boundary of the cylinder. As mentioned in the introduction, we have two types of inversion results. The first, Theorem 3, is really a corollary of one of the inversion formulas for circular means from the previous section.

Proof of Theorem [3. Let $u(x, t)$ to be the solution of the IVP (11), (2) in dimension two. Then by (11),

$$
u(p, t)=\partial_{t} \int_{0}^{t} \frac{(r \mathcal{M} f)(p, r)}{\sqrt{t^{2}-r^{2}}} d r .
$$

for $p \in S$. We can recover the circular means from $u$ by the standard method of inverting an Abel type equation. The details are not hard and may be found, for example, in [12]. The result is

$$
(\mathcal{M} f)(p, t)=\frac{2}{\pi} \int_{0}^{r} \frac{u(p, t)}{\sqrt{r^{2}-t^{2}}} d t
$$

Inserting (43) into the inversion formula (4) for $\mathcal{M}$ and applying Fubini's theorem, gives, for $x \in D$,

$$
\begin{aligned}
f(x) & =\frac{1}{2 \pi R_{0}} \Delta \int_{S} \int_{0}^{2 R_{0}}(r \mathcal{M} f)(p, r) \log \left|r^{2}-\right| x-\left.p\right|^{2} \mid d r d s(p) . \\
& =\frac{1}{R_{0} \pi^{2}} \Delta \int_{S} \int_{0}^{2 R_{0}} r \int_{0}^{r} \frac{u(p, t)}{\sqrt{r^{2}-t^{2}}} \log \left|r^{2}-\right| x-\left.p\right|^{2} \mid d t d r d s(p) \\
& =\frac{1}{R_{0} \pi^{2}} \Delta \int_{S} \int_{0}^{2 R_{0}} u(p, t) \int_{t}^{2 R_{0}} \frac{r}{\sqrt{r^{2}-t^{2}}} \log \left|r^{2}-\right| x-\left.p\right|^{2} \mid d r d t d s(p) \\
& =\frac{1}{R_{0} \pi^{2}} \Delta \int_{S} \int_{0}^{2 R_{0}} u(p, t) K(t,|x-p|) d t d s(p) .
\end{aligned}
$$


Since $u(p, t)=(\mathcal{W} f)(p, t)$, this is (12), with

$$
K(t, \bar{r}):=\int_{t}^{2 R_{0}} \frac{r}{\sqrt{r^{2}-t^{2}}} \log \left|r^{2}-\bar{r}^{2}\right| d r .
$$

The integral in (44) can be evaluated exactly. For the sake of completeness, we give the analytic expression. If we substitute $r=\sqrt{t^{2}+\xi^{2}}$ in (44), then $d r=(\xi / r) d \xi$ and thus

$$
\begin{aligned}
K(t, \bar{r}) & =\int_{0}^{\sqrt{4 R_{0}^{2}-t^{2}}} \log \left|\xi^{2}+\left(t^{2}-\bar{r}^{2}\right)\right| d \xi \\
& =\sqrt{4 R_{0}^{2}-t^{2}}\left(-2+\log \left|4 R_{0}^{2}-\bar{r}^{2}\right|\right)+\Gamma(t, \bar{r}),
\end{aligned}
$$

where

$$
\Gamma(t, \bar{r})= \begin{cases}\sqrt{\bar{r}^{2}-t^{2}} \log \frac{\sqrt{4 R_{0}^{2}-t^{2}}+\sqrt{\bar{r}^{2}-t^{2}}}{\sqrt{4 R_{0}^{2}-t^{2}}-\sqrt{\bar{r}^{2}-t^{2}}} & t<\bar{r}, \\ 2 \sqrt{t^{2}-\bar{r}^{2}} \arctan \sqrt{\frac{4 R_{0}^{2}-t^{2}}{t^{2}-\bar{r}^{2}}} & t>\bar{r} .\end{cases}
$$

For the second type of inversion formula, we start by deriving a representation of the formal adjoint $\mathcal{P}^{*}$, for $n=2$. For any continuous function $G(p, t)$ on $S \times[0, \infty)$ that has a small amount of decay as $t \rightarrow \infty$, by Fubini's theorem, we have

$$
\begin{aligned}
\langle\mathcal{P} f, G\rangle & =\int_{S} \int_{0}^{\infty}(\mathcal{P} f)(p, t) G(p, t) d t d s(p) \\
& =\frac{1}{2 \pi} \int_{S} \int_{0}^{\infty} G(p, t)\left(\int_{0}^{t} \frac{r}{\sqrt{t^{2}-r^{2}}} \int_{S^{1}} f(p+r \omega) d s(\omega) d r\right) d t d s(p) \\
& =\frac{1}{2 \pi} \int_{S} \int_{0}^{\infty}\left(\int_{0}^{t} \int_{S^{1}} \frac{f(p+r \omega)}{\sqrt{t^{2}-r^{2}}} r d r d S(\omega)\right) G(p, t) d t d s(p) \\
& =\frac{1}{2 \pi} \int_{S} \int_{0}^{\infty}\left(\int_{\mathbf{R}^{2}} \frac{f(y)}{\sqrt{t^{2}-|y-p|^{2}}} \chi(\{|y-p|<t\}) d y\right) G(p, t) d t d s(p) \\
& =\frac{1}{2 \pi} \int_{\mathbf{R}^{2}} f(y)\left(\int_{S} \int_{|y-p|}^{\infty} \frac{G(p, t)}{\sqrt{t^{2}-|y-p|^{2}}} d t d s(p)\right) f(y) d y \\
& =\left\langle f, \mathcal{P}^{*} G\right\rangle,
\end{aligned}
$$

where

$$
\left(\mathcal{P}^{*} G\right)(y):=\frac{1}{2 \pi} \int_{S} \int_{|y-p|}^{\infty} \frac{G(p, t)}{\sqrt{t^{2}-|y-p|^{2}}} d t d s(p) .
$$

The integral in (45) will be absolutely convergent for continuous $G$ provided that $G$ has a small amount of decay as $t \rightarrow \infty$, for example if $G(p, t)=\mathcal{O}\left(1 / t^{\alpha}\right)$, as $t \rightarrow \infty$, for some $\alpha>0$. 
Next, we note a differentiation formula for the fractional integral appearing in (11).

Proposition 3.1. Let $h$ be differentiable on $[0, \infty)$. Then, for $t>0$,

$$
\partial_{t} \int_{0}^{t} \frac{r h(r)}{\sqrt{t^{2}-r^{2}}} d r=\frac{1}{t} \int_{0}^{t} \frac{r\left(\partial_{r} r h\right)(r)}{\sqrt{t^{2}-r^{2}}} d r
$$

Proof. Making the change of variable $r=t \xi$ in the integral on the left we have to evaluate

$$
\partial_{t} \int_{0}^{1} \frac{\xi}{\sqrt{1-\xi^{2}}} t h(t \xi) d \xi
$$

Here differentiation under the integral yields $\int_{0}^{1} \frac{\xi}{\sqrt{1-\xi^{2}}}\left(t \xi h^{\prime}(t \xi)+h(t \xi)\right) d \xi$, which is equal to the expression on the right side after changing back to integration with respect to $r=t \xi$.

Proof of (13) in Theorem 4 for $n=2$. We compute $\left(\mathcal{P}^{*} t \partial_{t}^{2} \mathcal{P} f\right)(x)$ for smooth $f$ supported in $\bar{B}$ and $x \in B$. The function $t \partial_{t}^{2} \mathcal{P} f$ has decay of order $1 / t^{2}$ as $t \rightarrow \infty$, and so lies in the domain of $\mathcal{P}^{*}$. Using the definitions of $\mathcal{P}$ and $\mathcal{P}^{*}$, and relation (46),

$$
\begin{aligned}
\left(\mathcal{P}^{*} t\right. & \left.\partial_{t}^{2} \mathcal{P} f\right)(x) \\
& =\frac{1}{2 \pi} \int_{S} \int_{|x-p|}^{\infty} \partial_{t}^{2}\left(\int_{0}^{t} \frac{r(\mathcal{M} f)(p, r)}{\sqrt{t^{2}-r^{2}}} d r\right) \frac{t d t d s(p)}{\sqrt{t^{2}-|x-p|^{2}}} \\
& =\frac{1}{2 \pi} \int_{S} \int_{|x-p|}^{\infty} \partial_{t}\left(\frac{1}{t} \int_{0}^{t} \frac{r\left(\partial_{r} r \mathcal{M} f\right)(p, r)}{\sqrt{t^{2}-r^{2}}} d r\right) \frac{t d t d s(p)}{\sqrt{t^{2}-|x-p|^{2}}}
\end{aligned}
$$

Carrying out the differentiation in $t$ using the chain rule, using again (46), and combining terms, the last integral can be rewritten as

$$
\frac{1}{2 \pi} \int_{S} \int_{|x-p|}^{\infty}\left(\int_{0}^{r} \frac{r\left(\partial_{r} r \partial_{r} r \mathcal{M} f\right)(p, r)-r\left(\partial_{r} r \mathcal{M} f\right)(p, r)}{t \sqrt{t^{2}-|x-p|^{2}} \sqrt{t^{2}-r^{2}}} d r\right) d t d s(p) .
$$

Using the identity

$$
\partial_{r} r \partial_{r} r h-\partial_{r} r h=\partial_{r} r\left(\partial_{r} r h-h\right)=\partial_{r} r r \partial_{r} h=\partial_{r} r^{2} \partial_{r} h
$$

and applying Fubini's theorem $\left(\mathcal{P}^{*} t \partial_{t}^{2} \mathcal{P} f\right)(x)$ is in turn is equal to

$$
\frac{1}{2 \pi} \int_{S} \int_{0}^{\infty} r\left(\partial_{r} r^{2} \partial_{r} \mathcal{M} f\right)(p, r)\left(\int_{\max (|x-p|, r)}^{\infty} \frac{d t}{t \sqrt{t^{2}-|x-p|^{2}} \sqrt{t^{2}-r^{2}}}\right) d r d s(p) .
$$

The inner integral evaluates to

$$
\frac{1}{2 r|x-p|} \log \frac{r+|x-p|}{|r-| x-p||}
$$


giving

$$
\left(\mathcal{P}^{*} t \partial_{t}^{2} \mathcal{P} f\right)(x)=\frac{1}{4 \pi} \int_{S}\left(\int_{0}^{\infty}\left(\partial_{r} r^{2} \partial_{r} \mathcal{M} f\right)(p, r) \log \frac{r+|x-p|}{|r-| x-p||} d r\right) \frac{d s(p)}{|x-p|} .
$$

Treating the inner integral in principal value sense, and integrating by parts, it is equal to the limit as $\varepsilon \rightarrow 0$ of boundary terms

$$
\left[\left(r^{2} \partial_{r} \mathcal{M} f\right)(p, r) \log \frac{r+|x-p|}{|x-p|-r}\right]_{0}^{|x-p|-\varepsilon}+\left[\left(r^{2} \partial_{r} \mathcal{M} f\right)(p, r) \log \frac{r+|x-p|}{r-|x-p|}\right]_{|x-y|+\varepsilon}^{\infty}
$$

plus the term

$$
I_{\epsilon}:=-\int_{\mathbf{R}^{+} \backslash[|x-p|-\varepsilon,|x-p|+\varepsilon]}\left(r \partial_{r} \mathcal{M} f\right)(p, r) r \partial_{r} \log \frac{r+|x-y|}{|r-| x-p||} d r .
$$

Using that $\mathcal{M} f$ is smooth, flat at $r=0$, and of bounded support in $(0, \infty)$, the limit of the boundary terms is zero. Using the identity

$$
r \partial_{r} \log \frac{r+|x-p|}{|r-| x-p||}=-|x-p| \partial_{r} \log \left|r^{2}-\right| x-\left.p\right|^{2} \mid
$$

followed by another integration by parts, yields the sum of another pair of boundary terms and

$$
I_{\epsilon}=-|x-p| \int_{R^{+} \backslash[|x-p|-\varepsilon,|x-p|+\varepsilon]}\left(\partial_{r} r \partial_{r} \mathcal{M} f\right)(p, r) \log \left|r^{2}-\right| x-\left.p\right|^{2} \mid d r .
$$

The boundary terms again evaluate to zero as $\varepsilon \rightarrow 0$ while the integral $I_{\epsilon}$ converges to

$$
-|x-p| \int_{0}^{\infty}\left(\partial_{r} r \partial_{r} \mathcal{M} f\right)(p, r) \log \left|r^{2}-\right| x-\left.p\right|^{2} \mid d r .
$$

Inserting this into (47) and taking into account the support of $\mathcal{M} f$, gives

$$
\left(\mathcal{P}^{*} t \partial_{t}^{2} \mathcal{P} f\right)(x)=-\frac{1}{4 \pi} \int_{S} \int_{0}^{2 R_{0}}\left(\partial_{r} r \partial_{r} \mathcal{M} f\right)(p, r) \log \left|r^{2}-\right| x-\left.p\right|^{2} \mid d r d s(p) .
$$

In view of (15) of Theorem 1, (13) in Theorem 4 is proved, for $n=2$.

Proof of Theorem [5. Formula (15), for $n=2$, an easy corollary of the result just established. Indeed, for $f, g$ smooth with compact support in the closed disk of radius $R_{0}$, then

$$
\langle f, g\rangle=-\frac{2}{R_{0}}\left\langle\mathcal{P}^{*} t \partial_{t}^{2} \mathcal{P} f, g\right\rangle=-\frac{2}{R_{0}}\left\langle t \partial_{t}^{2} \mathcal{P} f, \mathcal{P} g\right\rangle
$$

which is (15) for $n=2$, due to the definition of the operator $\mathcal{P}$. 
In (15), the left hand side is symmetric in $f$ and $g$, while the right side is not. Thus there is a companion identity, reversing the roles of $u$ and $v$ on the right. Taking the difference gives the equation

$$
0=\int_{S} \int_{0}^{\infty} t\left(u_{t t} v-u v_{t t}\right) d t d s(p) .
$$

Integrating by parts (the boundary terms vanish) yields

$$
0=\int_{S} \int_{0}^{\infty}\left(u_{t} v-u v_{t}\right) d t d s(p)
$$

and another integration by parts proves

$$
0=\int_{S} \int_{0}^{\infty} u_{t} v d t d s(p)=\int_{S} \int_{0}^{\infty} u v_{t} d t d s(p) .
$$

Using this and one integration by parts in (15) establishes (16), which completes the proof of Theorem 5 for $n=2$. The extension to higher (even) dimensions follows almost word for word the proof from [5. Section 4.2], where the trace identities in odd dimensions greater than three were proved from the three dimensional case.

Proof of Theorem 4 for $n>2$. Reversing the chain of reasoning in (48) proves (13) in $L^{2}$ sense from (15). Similarly, (14) follows from (16). However, as both sides are continuous functions when $f$ is smooth, the formulas hold pointwise as well.

\section{Numerical results}

In the previous sections, we have established several exact inversion formulas to recover a function $f$ supported in a closed disc $\bar{D}$ from either its spherical means $\mathcal{M} f$ or the trace $\mathcal{W} f$ of the solution of the wave equation with initial data $(f, 0)$. However, those formulas require continuous data, whereas in practical applications only a discrete data set is available. For example, in thermoacoustic tomography (see Figure 1) only a finite number of positions of the line detectors and finite number of samples in time are feasible. In this section we derive discrete filtered back-projection (FBP) algorithms with linear interpolation in dimension two and present some numerical results.

The derived FBP algorithms are numerical implementations of discretized versions of (44)-(17) and (12)-(14) and the derivation of any of them follows the same line. We shall focus on the implementation of (5), assuming uniformly sampled discrete data

$$
F^{k, m}:=(\mathcal{M} f)\left(p^{k}, r^{m}\right), \quad(k, m) \in\left\{0, \ldots, N_{\varphi}\right\} \times\left\{0, \ldots, N_{r}\right\},
$$


where $p^{k}:=R_{0}\left(\cos \left(k h_{\varphi}\right), \sin \left(k h_{\varphi}\right)\right), r^{m}:=m h_{r}, h_{\varphi}:=2 \pi /\left(N_{\varphi}+1\right)$ and $h_{r}:=2 R_{0} / N_{r}$. In order to motivate the derivation of a discrete FBP algorithm based on (5), we introduce the differential operator $\mathcal{D}:=\partial_{r} r \partial_{r}$ and the integral operator

$$
\begin{gathered}
\mathcal{I}: C_{0}^{\infty}\left(S \times\left[0,2 R_{0}\right)\right) \rightarrow C^{\infty}\left(S \times\left[0,2 R_{0}\right)\right) \\
(\mathcal{I} G)(p, \bar{r}):=\int_{0}^{2 R_{0}} G(p, r) \log \left|r^{2}-\bar{r}^{2}\right| d r
\end{gathered}
$$

which both act in the second component, and the so called back-projection operator

$$
\begin{aligned}
\mathcal{B}: C^{\infty}\left(S \times\left[0,2 R_{0}\right)\right) & \rightarrow C^{\infty}(\bar{D}) \\
(\mathcal{B} G)(x) & :=\frac{1}{2 \pi R_{0}} \int_{S} G(p,|x-p|) d s(p) \\
& =\frac{1}{2 \pi} \int_{0}^{2 \pi} G(p(\varphi),|x-p(\varphi)|) d \varphi
\end{aligned}
$$

where $p(\varphi):=R_{0}(\cos \varphi, \sin \varphi)$. Therefore, we can rewrite (51) as

$$
f=(\mathcal{B I} \mathcal{D})(\mathcal{M} f) .
$$

In the numerical implementation the operators $\mathcal{B}, \mathcal{I}$, and $\mathcal{D}$ in (52) are replaced with finite dimensional approximation $\mathbf{B}, \mathbf{I}$ and $\mathbf{D}$ (as described below) and (52) is approximated by

$$
f\left(x^{i}\right) \approx f^{i}:=(\mathbf{B} \mathbf{I} \mathbf{D} \mathbf{~})^{i}, \quad i \in\{0, \ldots, N\}^{2} .
$$

Here $\mathbf{F}:=\left(F^{k, m}\right)_{k, m}$ with $F^{k, m}$ defined by (49), $x^{i}:=-\left(R_{0}, R_{0}\right)+i h_{x}$ with $i=\left(i_{1}, i_{2}\right) \in$ $\{0, N\}^{2}$ and $h_{x}:=2 R_{0} / N$. In the following $\mathbf{S}_{\varphi, r}$ and $\mathbf{S}_{x}$ denote the sampling operators that map $G \in C^{\infty}\left(S \times\left[0,2 R_{0}\right]\right)$ and $f \in C^{\infty}(\bar{D})$ onto its samples, $\mathbf{S}_{\varphi, r} G:=\left(G\left(p^{k}, r^{m}\right)\right)_{k, m}$ and $\mathbf{S}_{x} f:=\mathbf{f}:=\left(f\left(x^{i}\right)\right)_{i}$, where we set $f\left(x^{i}\right):=0$ if $x^{i} \notin \bar{D}$. Moreover, $|\cdot|_{\infty}$ denotes the maximum norm on either $\mathbf{R}^{\left(N_{\varphi}+1\right) \times\left(N_{r}+1\right)}$ or $\mathbf{R}^{(N+1) \times(N+1)}$.

1. The operator $\mathcal{D}$ can be written as $\partial_{r}+r \partial_{r}^{2}$. We approximate $\partial_{r} G$ with symmetric finite differences $\left(G^{k, m+1}-G^{k, m-1}\right) /\left(2 h_{r}\right), \partial_{r}^{2} G$ by $\left(G^{k, m+1}+G^{k, m-1}-2 G^{k, m}\right) / h_{r}^{2}$ and the multiplication operator $G \mapsto r G$ by point-wise discrete multiplication $\left(G^{k, m}\right)_{k, m} \mapsto$ $\left(r^{m} G^{k, m}\right)_{k, m}$. This leads to the discrete approximation

$$
\begin{aligned}
\mathbf{D} & : \mathbf{R}^{\left(N_{\varphi}+1\right) \times\left(N_{r}+1\right)} \rightarrow \mathbf{R}^{\left(N_{\varphi}+1\right) \times\left(N_{r}+1\right)}, \\
\mathbf{F} & \mapsto\left((\mathbf{D ~ G})^{k, m}:=\frac{1}{h_{r}}\left(\left(m+\frac{1}{2}\right) G^{k, m+1}+\left(m-\frac{1}{2}\right) G^{k, m-1}-2 m G^{k, m}\right)\right)_{k, m}
\end{aligned}
$$

where we set $G^{k,-1}:=G^{k, N_{r}+1}:=0$. The approximation of $\partial_{r}$ with symmetric finite differences is of second order and therefore $\left|\left(\mathbf{S}_{\varphi, r} \mathcal{D}-\mathbf{D} \mathbf{S}_{\varphi, r}\right) G\right|_{\infty} \leq C_{1} h_{r}^{2}$ for some constant $C_{1}$ which does not depend on $h_{r}$. 
2. Next we define a second order approximation to the integral operator $\mathcal{I}$. This is done by replacing $G\left(p^{k}, \cdot\right)$ in (50) by the piecewise linear spline $T^{k}[G]:\left[0,2 R_{0}\right] \rightarrow \mathbf{R}$ interpolating $G$ at the nodes $r^{m}$. More precisely,

$$
\mathbf{I}: \mathbf{R}^{\left(N_{\varphi}+1\right) \times\left(N_{r}+1\right)} \rightarrow \mathbf{R}^{\left(N_{\varphi}+1\right) \times\left(N_{r}+1\right)}: \quad \mathbf{G} \mapsto\left((\mathbf{I} \mathbf{G})^{k, m}\right)_{k, m}
$$

is defined by

$$
T^{k}[\mathbf{G}](r):=G^{k, m}+\frac{r-r^{m}}{h_{r}}\left(G^{k, m+1}-G^{k, m}\right), \quad r \in\left[r^{m}, r^{m+1}\right]
$$

and

$$
\begin{aligned}
(\mathbf{I} F)^{k, m} & :=\int_{0}^{2 R_{0}} T^{k}[\mathbf{G}](r) \log \left|r^{2}-\left(r^{m}\right)^{2}\right| d r \\
& =\sum_{m^{\prime}=0}^{N_{r}-1} G^{k, m^{\prime}}\left(\int_{r^{m^{\prime}}}^{r^{m^{\prime}+1}} \log \left|r^{2}-\left(r^{m}\right)^{2}\right| d r\right) \\
& +\sum_{m^{\prime}=0}^{N_{r}-1} \frac{G^{k, m^{\prime}+1}-G^{k, m^{\prime}}}{h_{r}}\left(\int_{r^{m^{\prime}}}^{r^{m^{\prime}+1}}\left(r-r^{m^{\prime}}\right) \log \left|r^{2}-\left(r^{m}\right)^{2}\right| d r\right) .
\end{aligned}
$$

For an efficient and accurate numerical implementation it is crucial that the integrals in (56) are evaluated analytically. In fact, by straight forward computation it can be verified that

$$
\begin{aligned}
(\mathbf{I} F)^{k, m} & =\sum_{m^{\prime}=0}^{N_{r}-1} a_{m^{\prime}}^{m} G^{k, m^{\prime}}+\frac{1}{h_{r}} \sum_{m^{\prime}=0}^{N_{r}-1} b_{m^{\prime}}^{m}\left(G^{k, m^{\prime}+1}-G^{k, m^{\prime}}\right), \\
a_{m^{\prime}}^{m} & :=\left[\left(r-r^{m}\right) \log \left|r-r^{m}\right|+\left(r+r^{m}\right) \log \left|r+r^{m}\right|-2 r\right]_{r=r^{m^{\prime}}}^{r^{m^{\prime}+1}}, \\
b_{m^{\prime}}^{m} & :=-r^{m^{\prime}} a_{m^{\prime}}^{m}+\frac{1}{2}\left[\left(r^{2}-\left(r^{m}\right)^{2}\right) \log \left|r^{2}-\left(r^{m}\right)^{2}\right|-r^{2}\right]_{r=r^{m^{\prime}}}^{r^{m^{\prime}+1}} .
\end{aligned}
$$

Moreover, using the fact that piecewise linear interpolation is of second order [15] and that $r \mapsto \log \left|r-\left(r^{m}\right)^{2}\right|$ is integrable, it can be readily verified that the approximation error satisfies $\left|\left(\mathbf{S}_{\varphi, r} \mathcal{I}-\mathbf{I} \mathbf{S}_{\varphi, r}\right) G\right|_{\infty} \leq C_{2} h_{r}^{2}$ with some constant $C_{2}$ independent of $h_{r}$.

3. Finally, we define a second order approximation to the back-projection (51). The discrete back-projection operator $\mathbf{B}: \mathbf{R}^{\left(N_{\varphi}+1\right) \times\left(N_{r}+1\right)} \rightarrow \mathbf{R}^{(N+1) \times(N+1)}$ is obtained by approximating (51) with the trapezoidal rule and piecewise linear interpolation (55) in the second variable,

$$
(\mathbf{B ~ G})^{i}:=\frac{1}{N_{\varphi}+1} \sum_{k=0}^{N_{\varphi}} T^{k}[\mathbf{G}]\left(\left|x^{i}-p^{k}\right|\right), \quad x^{i} \in D,
$$

and setting $(\mathbf{B ~ G})^{i}:=0$ for $x^{i} \notin D$. It is well known [15] that both linear interpolation in $r$ and the trapezoidal rule in $\varphi$ are second order approximations and therefore $\left|\left(\mathbf{S}_{x} \mathcal{B}-\mathbf{B} \mathbf{S}_{\varphi, r}\right) G\right|_{\infty} \leq C_{3} \max \left\{h_{r}^{2}, h_{\varphi}^{2}\right\}$ for some constant $C_{3}$. 


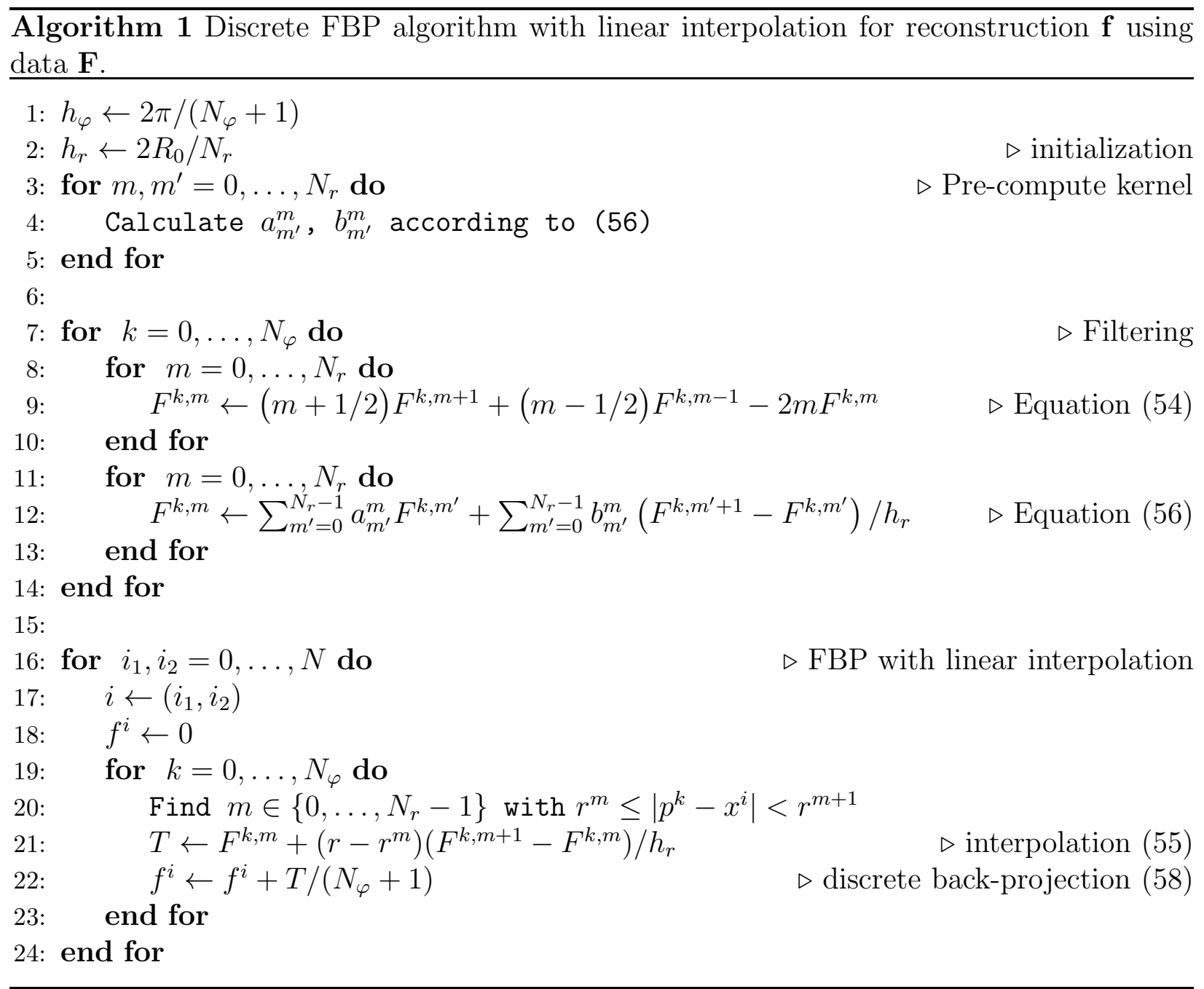

The discrete FBP algorithm is given by (53) with D, I, B defined in (54), (157), (58) and is summarized in Algorithm 1. Using $f\left(x^{i}\right)=\left(\mathbf{S}_{x} \mathcal{B} \mathcal{I} \mathcal{D} F\right)^{i}=\left(\mathbf{S}_{x} f\right)^{i}$ and $f^{i}=\left(\mathbf{B} \mathbf{I} \mathbf{D} \mathbf{S}_{\varphi, r} F\right)^{i}$, the discretization error $\left|f\left(x^{i}\right)-f^{i}\right|$ can be estimated as

$$
\begin{aligned}
\left|\left(\mathbf{S}_{x} \mathcal{B I} \mathcal{I}-\mathbf{B ~ I D ~ S}_{\varphi, r}\right) F\right|_{\infty} & \leq\left|\left(\mathbf{S}_{x} \mathcal{B}-\mathbf{B ~ S}_{\varphi, r}\right)(\mathcal{I} \mathcal{D} F)\right|_{\infty} \\
& +\left|\mathbf{B}\left(\mathbf{S}_{\varphi, r} \mathcal{I}-\mathbf{I} \mathbf{S}_{\varphi, r}\right)(\mathcal{D} F)\right|_{\infty} \\
& +\left|\mathbf{B} \mathbf{I}\left(\mathbf{S}_{\varphi, r} \mathcal{D}-\mathbf{D} \mathbf{S}_{\varphi, r}\right)(F)\right|_{\infty}
\end{aligned}
$$

Using the facts that $\mathbf{B}$ and $\mathbf{I}$ are bounded by some constant independent of $h_{r}$ and that the approximation of $\mathcal{D}, \mathcal{I}, \mathcal{B}$ with $\mathbf{D}, \mathbf{I}, \mathbf{B}$ are of second order, implies that

$$
\left|\mathbf{S}_{x} f-\mathbf{B} \mathbf{I D} \mathbf{F}\right|_{\infty} \leq C \max \left\{h_{r}^{2}, h_{\varphi}^{2}\right\},
$$

for some constant $C$ independent of $h_{r}, h_{\varphi}$. This shows that the derived FBP algorithm has second order accuracy (for exact data). 

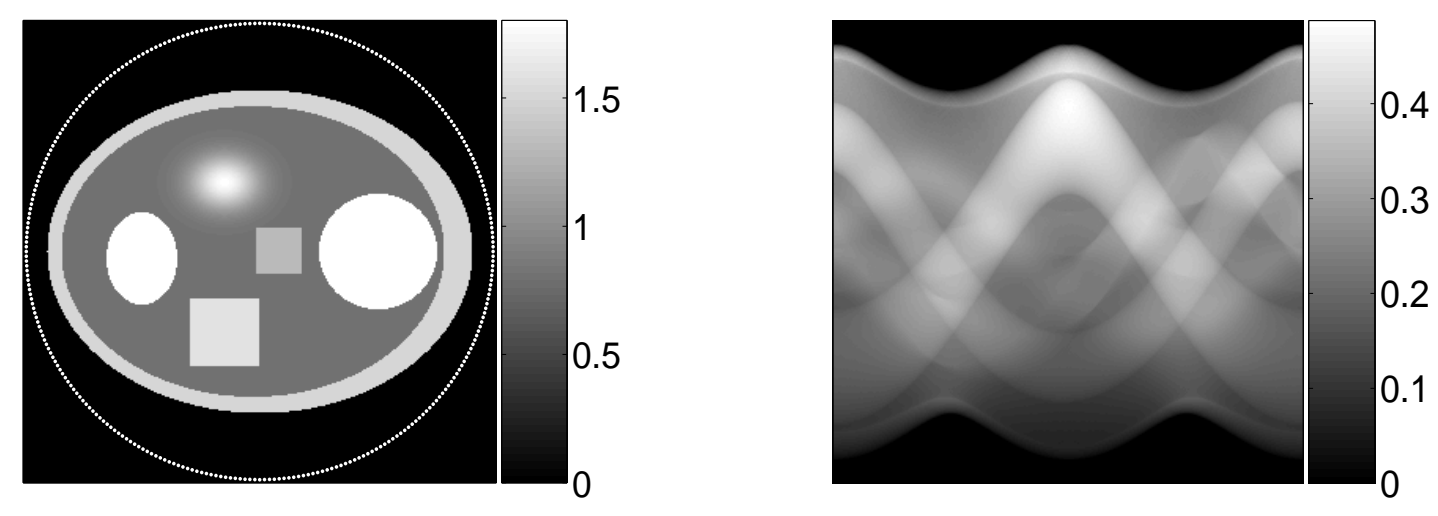

Figure 2: Imaging phantom and data. Left: Imaging phantom $f$ consisting of several characteristic functions and one Gaussian kernel. Right: Simulated data $F=\mathcal{M} f$.

In the numerical implementation, the coefficients in (154), are pre-computed and stored. Therefore the numerical effort of the evaluating (54) is $\mathcal{O}\left(N_{r}^{2} N_{\varphi}\right)$. Moreover, (54) requires $\mathcal{O}\left(N_{r} N_{\varphi}\right)$ operations and the discrete $\mathrm{FBP} \mathcal{O}\left(N^{2} N_{\varphi}\right)$, since for all $(N+1)^{2}$ reconstruction points $x^{i}$ we have to sum over $N_{\varphi}+1$ center locations on $S$. Hence, assuming $N \sim N_{r}$ and $N \sim N_{\varphi}$, Algorithm 1 requires $\mathcal{O}\left(N^{3}\right)$ operations and therefore has the same numerical effort as the classical FBP algorithm used in x-ray CT [12. Analogous to the procedure described above, discrete FBP algorithms were derived using equation (44), (6) for inverting $\mathcal{M}$ and (12) for inverting $\mathcal{W}$.

In the following we present numerical results of our FBP algorithms for reconstruction the phantom shown in the left picture in Figure 2, consisting of a superposition of characteristic functions and one Gaussian kernel. We calculated the data $\mathcal{M} f$ via numerical integration and the operator $\mathcal{W} f=\partial_{t} \mathcal{P} f$ using (11). Subsequently we added $5 \%$ uniformly distributed noise to $\mathcal{M} f$ and $10 \%$ uniformly distributed noise to $\mathcal{W} f$. The results for $N=N_{\varphi}=N_{r}=300$ using the algorithms based on (44), (5), (6) and (12) are depicted in Figures 3, 4and 5, All implementations show good results although no explicit regularization strategy is incorporated in order to the regularize the involved (mildly) ill posed numerical differentiation. In particular, (6) and (12) appear to be most insensitive to noise. However, for noisy data, the accuracy of FBP algorithms can be further improved by incorporating a regularizing strategy similar to that used in [8]. The derived identities in this article provide the mathematical foundation for further development of FBP algorithms for the inversion from spherical means and the inversion of the wave equation. 

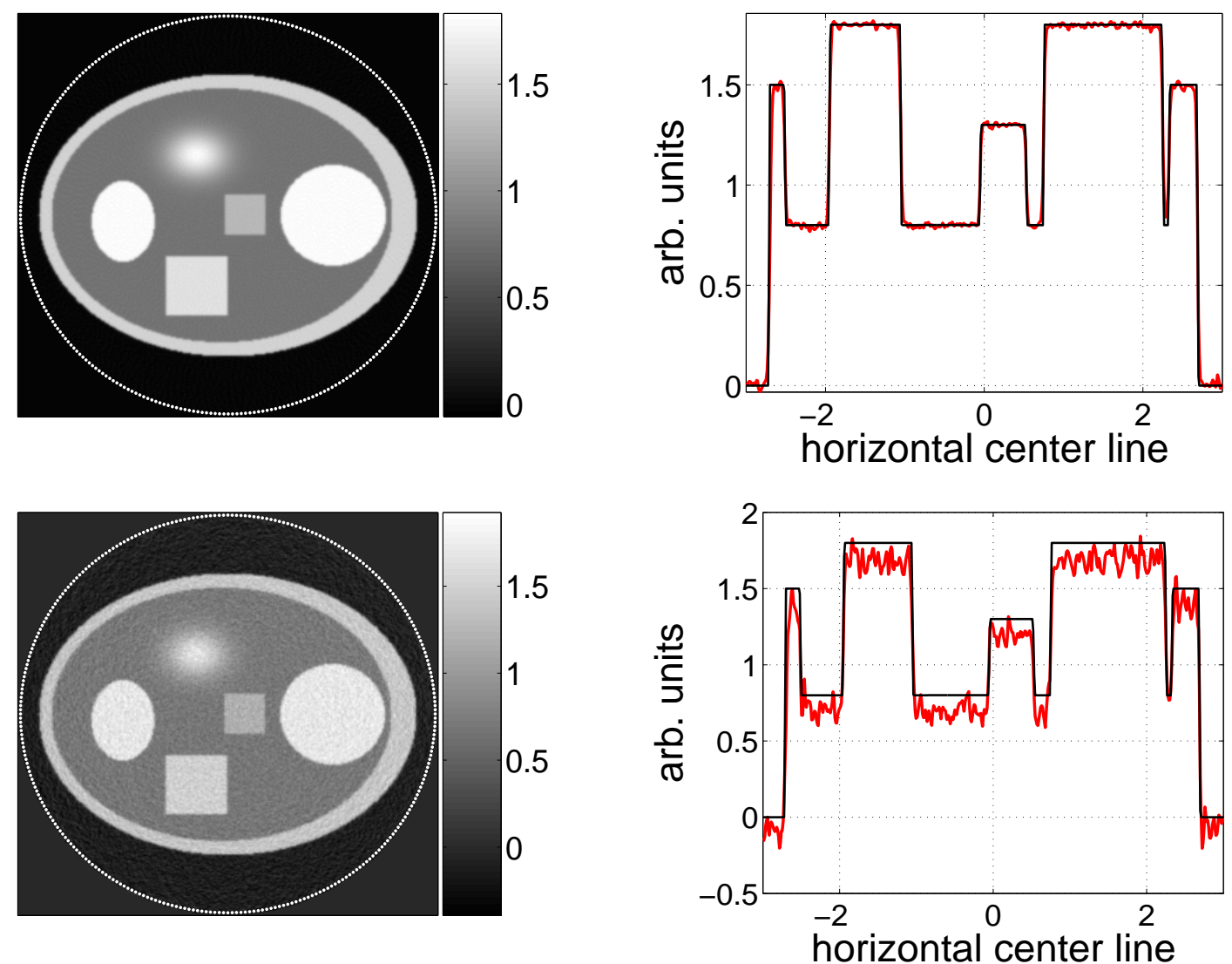

Figure 3: Numerical Reconstruction with Algorithm 1. Top: Reconstructions from simulated data. Bottom: Reconstructions from simulated data after adding 5\% uniformly distributed noise.

\section{References}

[1] M. Agranovsky, P. Kuchment, and E.T Quinto, Range descriptions for the spherical mean Radon transform., submitted (2006).

[2] G. Ambartsoumian and P. Kuchment, A range description for the planar circular Radon transform, SIAM J. Math. Anal. 38 (2006), 681-692.

[3] P. Burgholzer, C. Hofer, G. Paltauf, Haltmeier M., and O. Scherzer, Thermoacoustic tomography with integrating area and line detectors, IEEE Trans. Ultrason. Ferroelec. Freq. Contr. 52 (2005), 1577-1583.

[4] R. Courant and D. Hilbert, Methoden der mathematischen Physik, 4 ed., SpringerVerlag, Berlin, Heidelberg, New York, 1993. 

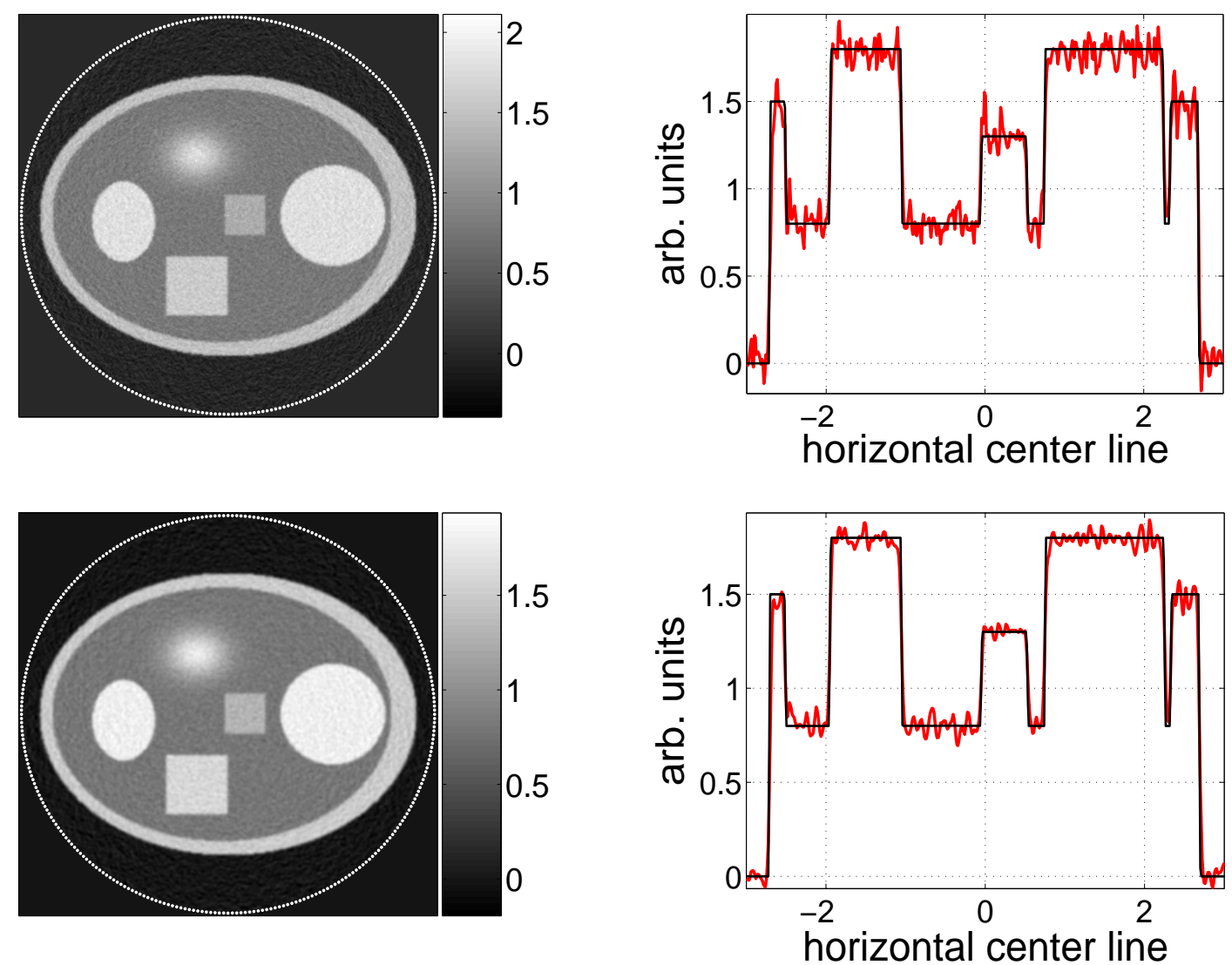

Figure 4: Numerical reconstruction from spherical means with $5 \%$ noise added. Top: Reconstruction using (44). Bottom: Reconstruction using (6)

[5] D. Finch, S.K. Patch, and Rakesh, Determining a function from its mean values over a family of spheres, SIAM J. Math. Anal. 35 (2004), 1213-1240.

[6] D. Finch and Rakesh, The range of the spherical mean value operator for functions supported in a ball, Inverse Problems 22 (2006), 923-938.

[7] M. Haltmeier and T. Fidler, Mathematical challenges arising in thermoacoustic computed tomography with line detectors, math.AP/0610155 (2006).

[8] M. Haltmeier, T. Schuster, and O. Scherzer, Filtered backprojection for thermoacoustic computed tomography in spherical geometry, Math. Meth. Appl. Sci. 28 (2005), no. 16, 1919-1937.

[9] R.A. Kruger, J.L. Kiser, D.R. Reinecke, G.A. Kruger, and K.D. Miller, Thermoacoustic optical molecular imaging of small animals, Molecular Imaging 2 (2003), 113-123. 

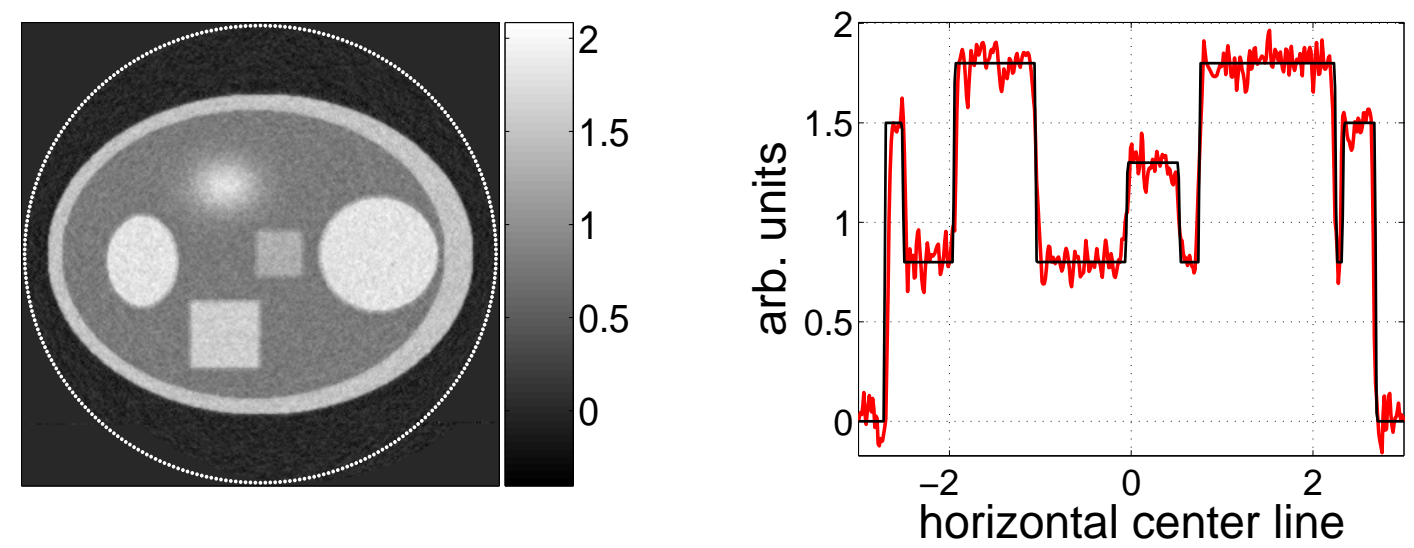

Figure 5: Numerical reconstruction using (12) from trace $\mathcal{W} f$ of the solution of the wave equation with $10 \%$ noise added.

[10] R.A. Kruger, K.D. Miller, H.E. Reynolds, W.L. Kiser, D.R. Reinecke, and G.A. Kruger, Breast cancer in vivo: contrast enhancement with thermoacoustic ct at 434 mhz-feasibility study, Radiology 216 (2000), 279-283.

[11] L. Kunyansky, Explicit inversion formulas for the spherical mean transform, arXiv:math.AP/0609341, Inverse Problems, to appear.

[12] F. Natterer, The mathematics of computerized tomography, Wiley, Chichester, 1986.

[13] S. J. Norton, Reconstruction of a two-dimensional reflecting medium over a circular domain: Exact solution, J. Acoust. Soc. Amer. 67 (1980), 1266-1273.

[14] G. Paltauf, R. Nuster, M. Haltmeier, and P. Burgholzer, Thermoacoustic computed tomography using a Mach-Zehnder interferometer as acoustic line detector, submitted (2006).

[15] A. Quateroni, R. Sacco, and F. Saleri, Numerical mathematics, Springer, 2000.

[16] X.D. Wang, G. Pang, Y.J. Ku, X.Y. Xie, G. Stoica, and L.-H.V. Wang, Noninvasive laser-induced photoacoustic tomography for structural and functional in vivo imaging of the brain, Nature Biotechnology 21 (2003), 803-806.

[17] M. Xu and L.-H.V. Wang, Photoacoustic imaging in biomedicine, Review of Scientific Instruments 77 (2006), 0411011-22. 